\title{
Cardiac status after long-term growth hormone replacement therapy in adult growth hormone deficient patients. \\ A single centre audit based on echocardiographic investigations before and during GH replacement therapy
}

Sissela Skoglund ${ }^{1}$, Ulla Feldt-Rasmussen ${ }^{1}$, Marianne Klose ${ }^{1}$ and Christian Hassager ${ }^{2}$ ${ }^{1}$ Department of Medical Endocrinology, Rigshospitalet, University Hospital of Copenhagen, Denmark ${ }^{2}$ Department of Cardiology, University Hospital of Copenhagen, Denmark

\section{Background and aim}

Growth hormone $(\mathrm{GH})$ is known as a hormone involved in regulation and influence of somatic growth but also of a long variety of metabolic pathways. GH exerts its effect in the body directly and indirectly, by stimulating insulin like growth factor-1 (IGF-1) production. Both $\mathrm{GH}$ and IGF-1 receptors are expressed in cardiac myocytes.

GH deficiency may develop deformities of cardiac structure as well as impaired systolic and diastolic functions. Further, earlier investigations indicate that IGF-1 excess has an hypertrophic effect on cardiac cells.

The aim of this study was to determine the effects of long-term $\mathrm{GH}$ treatment on heart anatomy and function among adult $\mathrm{GH}$ deficient patients.

\section{Patients and methods}

All GH deficient patients followed at Rigshospitalet with available echocardiography data at treatment baseline, 3-5 years and 8-10 years follow up were included. 25 naïve $\mathrm{GH}$ deficient patients i.e. patients who had never received $\mathrm{GH}$ therapy before, and 16 semi-naive patients i.e. patients previously on $\mathrm{GH}$ substitution therapy, but who had not received $\mathrm{GH}$ within 6 months before baseline evaluation.

Ultrasonic and biochemical data were collected retrospectively. Echocardiographic assessments were performed in accordance with the recommendations of the American Society of Echocardiography with focus on left ventricular structure and function.

\section{Results}

Table 1. Baseline characteristics of $N=41 \mathrm{GH}$ deficient patients. Results are expressed as $\mathrm{N}(\%)$ or mean \pm standard error of the mean if normally distributed, and otherwise as median (range).

\begin{tabular}{|c|c|c|}
\hline \multicolumn{2}{|c|}{ Baseline characteristics } & $\mathbf{N}(\%)$ \\
\hline \multicolumn{3}{|c|}{ Gender } \\
\hline - & Female & $16(39)$ \\
\hline - & Male & $25(61)$ \\
\hline \multicolumn{3}{|c|}{ Growth hormone deficiency onset } \\
\hline & - Adult-onset & $23(56)$ \\
\hline & - Childhood-onset & $18(44)$ \\
\hline \multicolumn{3}{|c|}{ Growth hormone therapy } \\
\hline & Naive & $25(61)$ \\
\hline & Semi-naive & $16(39)$ \\
\hline \multicolumn{3}{|c|}{ Etiology of pituitary disease } \\
\hline - & Craniopharyngioma & $3(7)$ \\
\hline - & Secreting pituitary adenoma & $3(7)$ \\
\hline - & Nonfunctioning pituitary adenoma & $9(22)$ \\
\hline - & Other extrasellar tumors & $10(25)$ \\
\hline - & Cranial irradiation due to malignancy outside the cranium & $1(2)$ \\
\hline - & Idiopathic & $1(2)$ \\
\hline - & Other & $14(35)$ \\
\hline IGF-I & & $-0.95 \pm 0.3$ \\
\hline Heig & $\mathrm{cm}$ & $171.9 \pm 1.8$ \\
\hline Wei & $\mathrm{t}, \mathrm{kg}$ & $80.9 \pm 2.6$ \\
\hline Bod & nass index, $\mathrm{kg} / \mathrm{m} 2$ & $27.3 \pm 0.7$ \\
\hline Bod & urface area, $\mathbf{m}^{2}$ & $1.9 \pm 0.0$ \\
\hline Syst & ic blood pressure, mmHg & $120 \pm 3$ \\
\hline Dias & lic blood pressure, $\mathrm{mmHg}$ & $77 \pm 2$ \\
\hline Tota & holesterol, mmol/L & $4.9 \pm 0.2$ \\
\hline LDL, & $\mathrm{mol} / \mathrm{L}$ & $3.2 \pm 0.2$ \\
\hline HDL, & $\mathrm{nmol} / \mathrm{L}$ & $1.3 \pm 0.1$ \\
\hline Trigl & erides, mmol/L & $1.0(0.3-6.4)$ \\
\hline Seru & Testosterone, $\mathrm{mmol} / \mathrm{L}$ (men, $\mathrm{N}=25$ ) & $17.5 \pm 2.2$ \\
\hline \multicolumn{3}{|c|}{ Other hormonal deficiency } \\
\hline & ionadal axis & $25(61)$ \\
\hline & Thyroid axis & $29(71)$ \\
\hline & Hypothalamic-pituitary-adrenal axis & $20(49)$ \\
\hline & Antidiuretic hormone axis & $7(17)$ \\
\hline
\end{tabular}

Table 2. IGF-1 standard deviation scores at follow-up compared to baseline in different groups. All numbers are expressed as mean \pm standard error of the mean Results are generated from a mixed model analysis. ${ }^{*} \mathrm{P}<0.05$

\begin{tabular}{lcrrr} 
IGF-1 standard deviation scores & N & \multicolumn{2}{c}{ Baseline } & \multicolumn{2}{c}{ 3-5 years } & \multicolumn{2}{c}{ 8-10 years } \\
All patients & 41 & $-0.9 \pm 0.3$ & $1.0^{*} \pm 0.4$ & $0.5^{*} \pm 0.3$ \\
Adulthood-onset total & 23 & $-1.48 \pm 0.29$ & $1.0^{*} \pm 0.4$ & $0.3^{*} \pm 0.4$ \\
Childhood-onset total & 18 & $-0.27 \pm 0.43$ & $0.9 \pm 0.6$ & $0.7^{*} \pm 0.5$ \\
Men & 25 & $-0.9 \pm 0.3$ & $1.4^{*} \pm 0.5$ & $0.9^{*} \pm 0.3$ \\
Women & 16 & $-1.0 \pm 0.5$ & $0.4^{*} \pm 0.5$ & $-0.0 \pm 0.5$ \\
Naive & 25 & $-1.4 \pm 0.3$ & $1.0^{*} \pm 0.4$ & $0.4^{*} \pm 0.4$ \\
Semi-naive & 16 & $-0.3 \pm 0.4$ & $0.8 \pm 0.6$ & $0.6 \pm 0.5$
\end{tabular}

\section{Conclusions}

Results from this study indicate that treatment duration of 8-10 years with $\mathrm{GH}$ replacement therapy in physiological doses in GH deficient patients appears not to be harmful, considering cardiac status. Verification of a positive effect of GH replacement on left ventricular mass index will, however, require a larger cohort of GHD patients.
Table 3. Left heart dimensions (A), systolic function (B) and diastolic function (C) at follow-up compared to baseline of $\mathrm{N}=41$ patients. Results are expressed as mean \pm standard deviation. Measurements from echocardiography. Results generated by a mixed model analysis. ${ }^{*} \mathrm{P}<0.05$.

\begin{tabular}{|c|c|c|c|}
\hline A. Cardiac structure & Baseline & $3-5$ years & $8-10$ years \\
\hline Inter ventricular septum diameter (mm) & $9.1 \pm 2.1$ & $\quad 9.2 \pm 2.2$ & $9.1 \pm 2.0$ \\
\hline Left ventricular internal dimension end diastole $(\mathrm{mm})$ & $45.7 \pm 8.5$ & $46.8 \pm 7.8$ & $45.6 \pm 8.8$ \\
\hline Left ventricular internal diameter end systole (mm) & $29.5 \pm 5.0$ & $30.9 \pm 5.9$ & $26.8 \pm 6.2$ \\
\hline Left ventricular posterior wall dimension $(\mathrm{mm})$ & $8.9 \pm 1.7$ & $9.4 \pm 1.8$ & $9.0 \pm 1.6$ \\
\hline Left ventricular mass (g) & $145.6 \pm 51.1$ & $151.4 \pm 52.9$ & $141.6 \pm 48.5$ \\
\hline Left ventricular mass index $(\mathrm{g} / \mathrm{m} 2)$ & $74.1 \pm 20.5$ & $76.6 \pm 21.3$ & $72.7 \pm 20.4$ \\
\hline \multicolumn{4}{|l|}{ B. Systolic function } \\
\hline Fractional shortening (\%) & $37.1 \pm 8.9$ & $34.0 \pm 9.0$ & $40.3 \pm 12.5$ \\
\hline \multicolumn{4}{|l|}{ C. Diastolic function } \\
\hline Mitral valve flow E/A ratio & $1.4 \pm 0.6$ & $1.3 \pm 0.5$ & $1.3 \pm 0.5$ \\
\hline Mitral valve deceleration time $(s)$ & $0.19 \pm 0.07$ & $0.19 \pm 0.04$ & $0.19 \pm 0.05$ \\
\hline
\end{tabular}

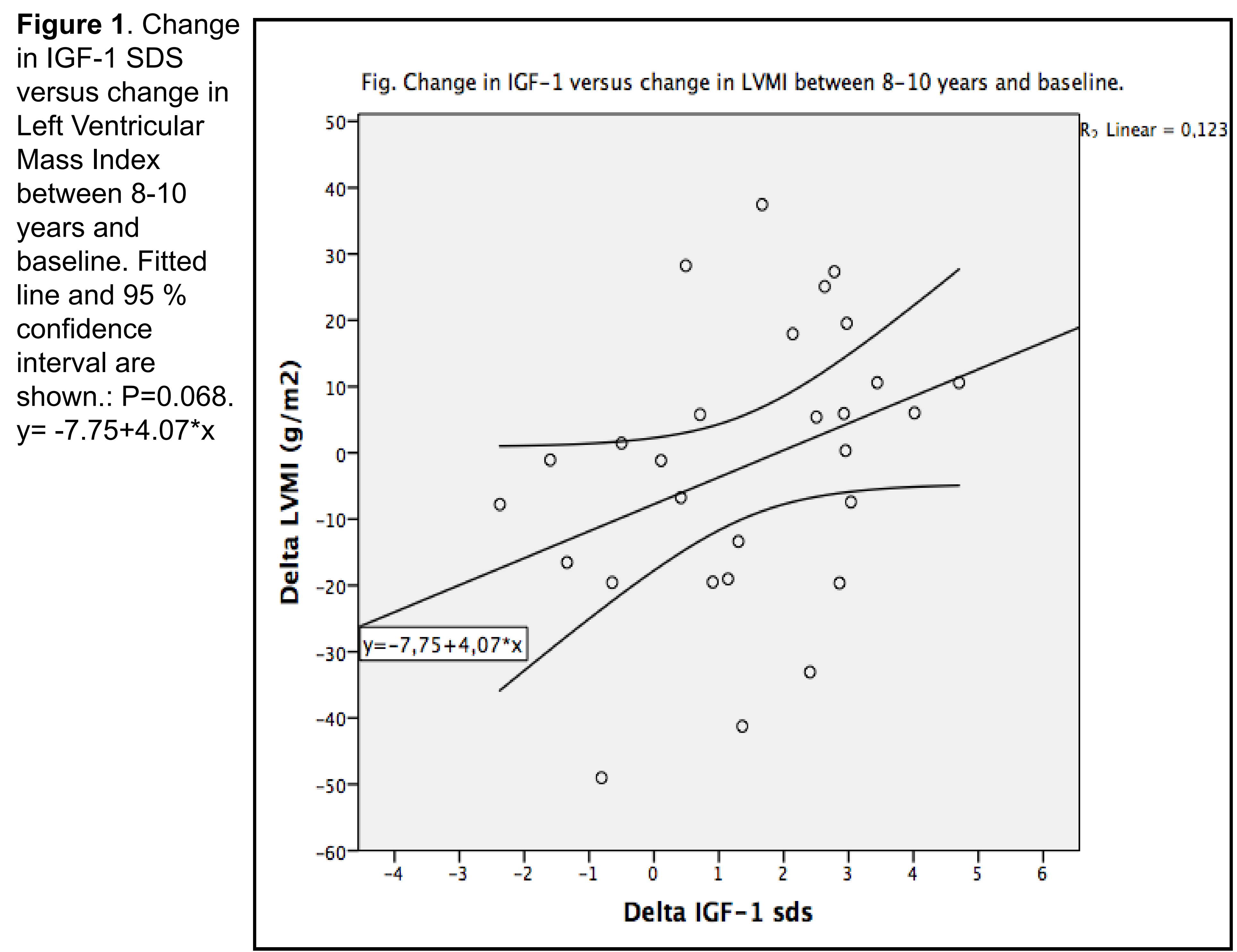

- No significant difference was observed in cardiac structure, nor in cardiac systolic or diastolic function during long term GH therapy.

- When dividing the patient cohort into subgroups a decrease in diastolic function was observed among naïve and adulthood-onset patients.

- A non-significant positive correlation was observed between change in IGF-1 SDS versus change in LVMI, at 3-5 years and 8-10 years follow-up. 